J. Clin. Chem. Clin. Biochem.

Vol. 16, 1978, pp. 519-524

\title{
Hereditäre Koproporphyrie in der Bundesrepublik Deutschland
}

\author{
Von M. Doss, R. v. Tiepermann und F. Verspohl
}

Fach Klinische Biochemie im Fachbereich Humanmedizin der Philipps-Universität, Marburg an der Lahn

In Kooperation mit der

Neurologischen Klinik (Leiter: Prof. Dr. W. Dorndorf)

im Zentrum für Neurologie des Klinikums der Justus-Liebig-Universität Gießen,

der Abteilung für Nieren- und Hochdruckkranke (Dir.: Prof. Dr. K. D. Bock)

der Medizinischen Klinik und Poliklinik des Universitätsklinikums Essen,

der Medizinischen Klinik (Chefarzt PD Dr. K.-P. Littmann)

des Stad tkrankenhauses Holwedestraße Braunschweig

und der Klinik Wingertsberg der BfA (Chefarzt Dr. H. Stolz)

in Bad Homburg v. d. H.

(Eingegangen am 23. Mai/10. August 1978)

Herrn Professor Dr. Ernst Schütte zum 70. Geburtstag gewidmet

Zusammenfassung: Die hereditäre Koproporphyrie gehört mit der akuten intermittierenden Porphyrie und Porphyria variegata zu den akuten hepatischen Porphyrien, denen eine Störung der Regulation der Hämsynthese zugrundeliegt („molekulare Regulationskrankheiten“). Hier werden die ersten vier Beobachtungen einer hereditären Koproporphyrie in der Bundesrepublik Deutschland beschrieben sowie ihre Differentialdiagnose und pathobiochemische Genese dargelegt. Es handelt sich um vier Patientinnen, von denen zwei unter dem Verdacht auf eine akute intermittierende Porphyrie untersucht wurden. Die charakteristische Konstellation der Porphyrinvorläufer- und Porphyrinausscheidung im Urin sowie der Porphyrinausscheidung im Stuhl mit der Dominanz von Koproporphyrin-Isomer-III führte zur Diagnose ,hereditäre Koproporphyrie‘. Die Bestimmung der Uroporphyrinogen-Synthase in den Erythrocyten wurde zur weiteren differentialdiagnostischen Abgrenzung herangezogen, da auch bei akuter intermittierender Porphyrie die Koproporphyrin-Isomer-III-Ausscheidung im Ựin, aber auch im Stuhl, erhöht und im Porphyrinprofil dominant sein kann. Die klinische Symptomatik war derjenigen einer akuten intermittierenden Porphyrie analog.

Prophylaktische Maßnahmen wie bei der akuten intermittierenden Porphyrie sind die wichtigsten ,therapeutischen ${ }^{\circ}$ Konsequenzen in den Latenzphasen.

Die vorwiegend historisch und symptomatologisch deskriptiv verstehbaren Begriffe ,akute intermittierende Porphyrie“, „hereditäre Koproporphyrie“" und „Porphyria variegata“ sollten dem pathobiochemisch, diagnostisch und klinisch kennzeichnenderen Terminus „akute hepatische Porphyrie“ zumindest nachgeordnet werden, da die Ubergänge zwischen diesen Formen der akuten hepatischen Porphyrien, die sämtlich eine ,akute intermittierende Manifestation' entwickeln können, fließend sind. Die Untersuchung der Porphyrinausscheidung im Stuhl sollte bei den akuten hepatischen Porphyrien neben der Erhebung des Urinporphyrinbiochemogramms in allen Fällen durchgefuhrt werden.

\section{Hereditary coproporphyria in the Federal Republic of Germany}

Summary: Hereditary coproporphyria belongs to the acute hepatic porphyrias - along with acute intermittent porphyria and porphyria variegata - which are all based on disturbances in heme biosynthesis ("molecular regulation diseases"). The first four cases of hereditary coproporphyria in the Federal Republic of Germany are presented pointing out their differential diagnosis and pathobiochemical genesis. Samples of two of the four female patients were investigated for acute intermittent porphyria. The characteristic renal porphyrin precursor and porphyrin elimination and fecal porphyrin excretion with dominance of coproporphyrin isomer III led to the diagnosis "hereditary coproporphyria". Uroporphyrinogen synthase was determined in the erythrocytes for further delimitation, since not only raised but even dominant coproporphyrinogen isomer III elimination in urine and stool are observed in acute intermittent porphyria too. Clinical signs were analogous to acute intermittent porphyria. 
The main "therapeutical" consequences during the latent phases are prophylactical measures which are the same as in acute intermittent porphyria.

Instead of the historic and symptomatic descriptive terms "acute intermittent porphyria", "hereditary coproporphyria" and "porphyria variegata", the pathobiochemically diagnostically and clinically more characteristic term "acute hepatic porphyria" should be used because of the transitions between these forms of acute hepatic porphyrias all of which can produce an "acute intermittent manifestation". In acute hepatic porphyrias urinary and fecal analyses of porphyrin excretion should be performed.

\section{Einfuihrung}

Die hereditäre Koproporphyrie kommt unter den akuten hepatischen Porphyrien (akute intermittierende Porphyrie, Porphyria variegata and hereditäre Koproporphyrie) am seltensten vor. Sie wird ebenfalls, wie die akute intermittierende Porphyrie und die Porphyria variegata, autosomal dominant vererbt und vermag ein gleich-schweres akutes klinisches Syndrom wie die akute intermittierende Porphyrie mit abdominalen, neurologischen und psychischen sowie cardiovaskulären Symptomen zu entwickeln (1). Als primärer genetischer Enzymdefekt wurde eine verminderte Aktivität der Koproporphyrinogen-Oxidase in Leukocyten (2), Lymphocyten (3) und Hautfibroblastenkulturen (4) erkannt. Klinisch-biochemisches Leitsymptom ist die hohe Koproporphyrin-Isomer-III-Ausscheidung in Stuhl und Urin. Da zwischen den hereditären akuten hepatischen Porphyrien fließende klinische und pathobiochemische Übergänge bestehen $(1,5)$, kann von klinischer Seite lediglich Verdacht auf eine ,akute intermittierende Porphyrie" geäußert werden; aber auch das Urinprofil der Porphyrinvorläufer und Porphyrine läßt nicht immer eine eindeutige Klärung zwischen akuter intermittierender Porphyrie, Porphyria variegata und hereditärer Koproporphyrie, insbesondere in den latenten Phasen,.zu. Die Differenzierung der Porphyrine im Stuhl wird hier entscheidend sein.

Die hereditäre Koproporphyrie ist in erster Linie eine molekulare Regulationskrankheit (5), da ihr primär eine Störung der Rückkopplungskontrolle des Häms auf die $\delta$-Aminolävulinsäure-Synthase in der Leber zugrundeliegt, deren Aktivität in der Leber während der akuten Attacke erhöht ist (6). Die hereditäre Koproporphyrie hat mit den anderen beiden akuten hepatischen Porphyrien auch die erhöhte Pharmakasensibilität, das Vorkommen in verschiedenen Phasen (genetische Phase, Latenzphasen und klinische Manifestation) sowie analoge therapeutische Erfordernisse gemeinsam $(5,7)$.

Die methodische Entwicklung differentialdiagnostischer Kriterien hat nicht nur zu einer relativ großen Zahl diagnostizierter Fälle und Genträger mit akuter intermittierender Porphyrie geführt - wir überblicken z. Zt. über 150 Fälle und zum Teil deren Familien -, sondern auch das Vorkommen der Porphyria variegata in Deutschland gesichert (8). Die vorliegende Arbeit über die ersten in Deutschland diagnostizierten Patienten mit hereditärer Koproporphyrie stellt einen Beitrag zur Differentialdiagnose der akuten hepatischen Porphyrien dar.

In rückblickender Umdeutung ist der von Hijmans von den Bergh et al. im Jahre 1928 publizierte „Fall von kongenitaler Porphyrinurie mit Koproporphyrin im Harn und Stuhl" (9a) wahrscheinlich als Erstbeschreibung einer hereditären Koproporphyrie zu würdigen; aus den Exkreten des Patienten isolierte und kristallisierte Hans Fischer Koproporphyrin-Isomer III. Als „idiopathic coproporphyrinuria" haben Watson und Mitarbeiter diese hepatische Porphyrie im Jahre 1949 bezeichnet ( $9 \mathrm{~b})$. Dem folgten weitere Berichte über das Vorkommen der hereditären Koproporphyrie aus England (10), Schweden (11), Dänemark $(12,13)$, Südafrika (14) und Frankreich (15). Die Manifestation einer hereditären Koproporphyrie kann durch orale Kontrazeptiva induziert werden (16). Aus einer englischen Studie über 111 Fälle geht hervor, daß 35\% der Patienten mit hereditärer Koproporphyrie eine akute Attacke entwickelten (2). Während der akuten metabolischen Krise werden auch große Mengen von $\delta$ Aminolävulinsäure und Porphobilinogen, der beiden Porphyrinvorläufer, im Urin ausgeschieden - analog zu den anderen akuten hepatischen Porphyrien. Die Verwechslung mit einer akuten intermittierenden Porphyrie liegt daher bei orientierender Diagnostik nahe. Im englischen Krankengut traten bei etwa 30\% der Fälle eine lichtinduzierte Photosensitivität während der akuten Attacke auf (2). Dieser Befund kann zur klinischen Abgrenżung einerseits gegenüber der akuten intermittierenden Porphyrie, die obligat ohne Hautsymptome verläuft, und andererseits gegenüber der Porphyria variegata herangezogen werden, bei der Hautsymptome auch in den Latenzphasen bestehen. Jedoch zeigten die in Deutschland beobachteten Porphyria variegata-Patienten in der Hälfte der Fälle keine Hautsymptome (8), und bei den bislang beobachteten Patienten mit heredițärer Koproporphyrie konnten Hautsymptome bis auf eine Ausnahme nicht festgestellt werden. Wahrscheinlich tritt die hereditäre Koproporphyrie in Mitteleuropa vorwiegend im klinischen Gewand der akkuten intermittierenden Porphyrie auf. 


\section{Patienten und Untersuchungsmethoden}

Die Untersuchung auf Porphyrinvorläufer und Porphyrine erfolgte entweder bei gezieltem klinischen Verdacht auf eine akute hepatische Porphyrie (Tab. 1 und 4) oder zum Ausschluß einer Porphyrie bzw. Porphyrinstoffwechselstörung (Tab. 2 und 5). Bei der Familienuntersuchung der Patientin M. T. (Tab. 2) wurde eine Schwester mit einer hereditären Koprophorphyrie in der Latenzphase entdeckt (Tab. 3). Die oberen Normgrenzen der Metabolite sind in Tabelle 2 angegeben. Die Legenden der Tabellen enthalten die wich tigsten anamnestischen und klinischen Fakten.

Porphyrine im Urin und Stuhl wurden dünnschichtchromatographisch (17), Porphyrinvorläufer $\delta$-Aminolävulinsäure und Porphobilinogen ionenaustauschchromatographisch analysiert (18). Die Bestimmung der Koproporphyrin-Isomere erfolgte ebenfalls dünnschich tchromatographisch in mehreren Schritten (19). Die Aktivität der Uroporphyrinogen-Synthase wurde in den Ery throcy ten bestimmt (20).

\section{Ergebnisse und Diskussion}

Die Differentialdiagnose der hereditären Koproporphyrie gegenüber den anderen akuten hepatischen Porphyrien, der akuten intermittierenden Porphyrie und der Porphyria variegata, beruht auf der simultanen Untersuchung der Porphyrinvorläufer- und Porphyrinausscheidung im Urin sowie der Porphyrinausscheidung im Stuhl (21). Aufgrund der fließenden biochemischen Ubergänge zwischen den akuten hepatischen Porphyrien kann die Bestimmung der Aktivität der Uroporphyrinogen-Synthase ein zusätzliches wichtiges differentialdiagnostisches Kriterium sein. Diese Untersuchung ist in jenen Fällen wichtig, bei denen neben einer hohen Porphyrinvorläuferausscheidung Koproporphyrin als dominante Komponente im Urin auftritt und die Koproporphyrinausscheidung auch im Stuhl erhöht ist (1).
Wenn die Porphyrinausscheidung im Stuhl bei klinisch manifester akuter hepatischer Porphyrie unter $0,5 \mathrm{mg} / \mathrm{g}$ (obere Normgrenze siehe Tab. 2) liegt und die Aktivität der Uroporphyrinogen-Synthase um etwa $40 \%$ gegenüber den Kontrollen erniedrigt ist, handelt es sich um eine akute intermittierende Porphyrie (22).

Bei den vier Patientinnen mit hereditärer Koproporphyrie mit klinischer Symptomatik (Tab. 1, 2, 4 und 5) lag die Ausscheidung von $\delta$-Aminolävulinsäure dreimal im oberen Normbereich (Tab. 1, 4 und 5). Gleichzeitig war in diesen Fällen die PorphobilinogenAusscheidung entweder normal (Tab. 4) und grenzwertig (Tab. 5) oder mäßiggradig erhöht (Tab. 1). Nur im Falle einer schweren Landry-Paralyse bestand eine hohe Porphyrinvorläuferausscheidung (Tab. 2) neben einer extrem hohen Porphyrinausscheidung mit etwa $10 \mu \mathrm{mol} \mathrm{Koproporphyrin} \mathrm{im} \mathrm{24-h-Harn.} \mathrm{Hier} \mathrm{wurde}$ die Porphyrinausscheidung im Urin und Stuhl nicht zu Beginn der Entwicklung der schweren klinischen Symptomatik erfaßt, sondern erst im fortgeschrittenen Stadium (Guillain-Barré-Syndrom). Von besonderem Interesse ist hier die zunächst nahezu gleichgroße, stark erhöhte Ausscheidung von Kopro- und Protoporphyrin im Stuhl. Die hohen Konzentrationen der Porphyrine in Stuhl und Plasma bedingten eine direkte Fluoreszenz dieser Proben im langwelligen UV-Licht. Unter hochdosierter Glucose-Behandlung ging die Porphyrin- und Porphyrinvorläuferausscheidung sowohl im Urin als auch im Stuhl zurück. Das Verhältnis von Kopro- zu Protoporphyrin veränderte sich unter der Therapie auf etwa 2:1 (Koproporphyrin etwa 4 und Protoporphyrin etwa $2 \mu \mathrm{mol} / \mathrm{g}$ ) und hat somit die Diagnose hereditäre Koproporphyrie weiter gestützt. Der klinischerseits durchgefuhrte Watson-Schwarz-Test war negativ; auf-

Tab. 1. Porphyrinkonstellationen einschließlich der Porphyrinvorläufer im Urin und Stuhl bei einer 38jährigen Patientin (G. L.): Hereditäre Koproporphyrie.

Seit mehreren Jahren Schmerzen im Abdomen, Rücken und in der ,Herzgegend', Kopfschmerzen, Schwindelgefühl, Nervosität und Tachykardie. Klinischer Verdacht: akute intermittierende Porphyrie.

\begin{tabular}{|c|c|c|c|c|}
\hline & $\begin{array}{l}\text { Urin } \\
\text { (nmol/24 h) }\end{array}$ & (nach Therapie) & $\begin{array}{l}\text { Faeces } \\
(\mathrm{nmol} / \mathrm{g})\end{array}$ & (nach Therapie) \\
\hline $\begin{array}{l}\text { Uroporphyrin } \\
\text { Heptacarboxy porphyrin } \\
\text { Hexacarboxyporphyrin } \\
\text { Pentacarboxyporphyrin } \\
\text { Koproporphyrin } \\
\text { Tricarboxyporphyrin } \\
\text { Dicarboxy porphyrin } \\
\text { (Protoporphyrin) }\end{array}$ & $\begin{array}{c}102 \\
56 \\
35 \\
166 \\
1519(93 \% \text { III) } \\
121 \\
67\end{array}$ & $\begin{array}{c}76 \\
15 \\
13 \\
26 \\
209(92 \% \text { III }) \\
18 \\
5\end{array}$ & $\begin{array}{c}13 \\
9 \\
4 \\
27 \\
3133(95 \% \text { III) } \\
16 \\
481\end{array}$ & $\begin{array}{c}7 \\
5 \\
3 \\
24 \\
1939 \text { (95\% III) } \\
7 \\
50\end{array}$ \\
\hline Gesamt-Porphyrine & $\begin{array}{l}2066 \\
(\mu \mathrm{mol} / 24 \mathrm{~h})\end{array}$ & 362 & 3683 & 2035 \\
\hline 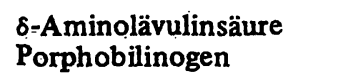 & $\begin{array}{l}39 \\
20\end{array}$ & $\begin{array}{r}24 \\
4\end{array}$ & & \\
\hline
\end{tabular}

Uroporphyrinogensy nthase in den Ery throcyten
(zwei Blutproben zu verschiedenen Zeiten) $61(93) \quad 65(101) \quad((\mu \mathrm{mol} / \mathrm{h} \cdot 1$ Erythrocyten (\% der Kontrollen)). 
Tab. 2. Hereditäre Koproporphyrie bei einer 50jährigen Patientin (M. T.) mit Tetraplegie, Ileus und respiratorischer Insuffizienz (Landry-Paralyse), Tachykardie und Hypokaliämie. Dem akuten Syndrom gingen eine Medikation mit Analgetica (Pyrazolon und Phenylbutazon) und Sulfonamiden sowie Narkosemaßnahmen (Barbiturate) voraus (Ureterolithiasis). Zur Abklärung des „Guillain-Barrè-Syndroms" wurde die Porphyrie-Diagnostik veranlaß t.

\begin{tabular}{|c|c|c|c|c|c|c|}
\hline & $\begin{array}{l}\text { Urin } \\
\text { (nmol/24 h) }\end{array}$ & $\begin{array}{l}\text { obere } \\
\text { Normgrenze }\end{array}$ & $\begin{array}{l}\text { Faeces } \\
(\mathrm{nmol} / \mathrm{g})\end{array}$ & $\begin{array}{l}\text { obere } \\
\text { Normgrenze }\end{array}$ & $\begin{array}{l}\text { Plasma } \\
\text { (nmol/l) }\end{array}$ & $\begin{array}{l}\text { obere } \\
\text { Norm- } \\
\text { grenze }\end{array}$ \\
\hline $\begin{array}{l}\text { Uroporphyrin } \\
\text { Heptacarboxyporphyrin } \\
\text { Hexacarboxy porphyrin } \\
\text { Pentacarboxy porphyrin } \\
\text { Koproporphyrin } \\
\text { Tricarboxyporphyrin } \\
\text { Dicarboxyporphyrin } \\
\text { (Protoporphyrin) }\end{array}$ & $\begin{array}{l}758 \\
251 \\
197 \\
899 \\
9406(84 \% \text { III) } \\
261 \\
233\end{array}$ & $\begin{array}{r}29 \\
4 \\
3 \\
6 \\
119(83 \% \text { III }) \\
2 \\
1\end{array}$ & $\begin{array}{l}92 \\
155 \\
74 \\
463 \\
1890(86 \% \text { III }) \\
440 \\
1821\end{array}$ & 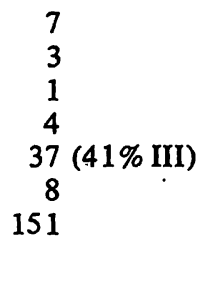 & $\begin{array}{l}81 \\
37\end{array}$ & $\begin{array}{l}0,2 \\
0,8\end{array}$ \\
\hline Gesamt-Porphyrine & $\begin{array}{l}12005 \\
(\mu \mathrm{mol} / 24 \mathrm{~h})\end{array}$ & & 4935 & . & - & \\
\hline $\begin{array}{l}\delta \text {-Aminolävulinsäure } \\
\text { Porphobilinogen }\end{array}$ & $\begin{array}{l}229 \\
307\end{array}$ & $\begin{array}{r}49 \\
8\end{array}$ & & & & \\
\hline
\end{tabular}

Uroporphyrinogensynthase in den Erythrocyten ( $\mu \mathrm{mol} / \mathrm{h} \cdot 1$ Ery throcyten (\% der Kontrollen)) 80 (127)

grund der hohen Diurese lag die Konzentration des Porphobilinogen bei nur etwa $45 \mu \mathrm{mol} / 1$. Urin- und Stuhluntersuchungen bei der Schwester der Patientin führten zur Diagnose einer hereditären Koproporphyrie in der kompensierten Latenzphase (Tab. 3).

Man muß bei der Diagnostik akuter hepatischer Porphyrien vom Typ der Porphyria variegata und der hereditären Koproporphyrie in Betracht ziehen, daß die Porphyrinvorläuferausscheidung meist nur zu Beginn der akuten Krise extreme Werte wie bei der akuten intermittierenden Porphyrie erreicht, danach aber

Tab. 3. Hereditäre Koproporphyrie in der kompensierten Latenzphase bei der 56jährigen Schwester (M. M.) der Patientin M. T. (s. Tab. 2). Ein akutes Syndrom mit kolikartigen Oberbauchschmerzen, Tetraplegie und Lähmung der A temmuskulatur war vorausgegangen und als akute in termittierende Porphyrie diagnostiziert worden.

\begin{tabular}{lcc}
\hline & $\begin{array}{l}\text { Urin } \\
\text { (nmol/24 h) }\end{array}$ & $\begin{array}{l}\text { Stuhl } \\
\text { (nmol/g) }\end{array}$ \\
\hline Uroporphyrin & 105 & 7 \\
Heptacarboxyporphyrin & 32 & 3 \\
Hexacarboxy porphyrin & 5 & 3 \\
Pentacarboxyporphyrin & 19 & 13 \\
Koproporphyrin & 153 & 105 \\
Tricarboxyporphyrin & 3 & 3 \\
Dicarboxyporphyrin & $<1$ & 101 \\
$\quad$ (Protoporphyrin) & & \\
Gesamt-Porphyrine & 318 & 235 \\
& & \\
& $(\mu \mathrm{mol} / 24 \mathrm{~h})$ & \\
8-Aminolävulinsäure & 26,7 & \\
Porphobilinogen & 1,5 & \\
\hline
\end{tabular}

schneller abfällt als bei der akuten intermittierenden Porphyrie. Die Porphyrinausscheidung bleibt indes extrem erhöht. Deshalb können Suchteste auf Porphobilinogen zur Entdeckung einer hereditären Koproporphyrie versagen. Bei der vergleichenden Betrachtung der klinischen und biochemischen Daten der vier Patientinnen mit hereditärer Koproporphyrie fällt eine gewisse Abhängigkeit $\mathrm{zwischen} \mathrm{der} \mathrm{Por-}$ phyrinausscheidung im Urin und der klinischen Symptomatik auf. Nach Therapie ist der Rückgang der Por: phyrinausscheidung im Urin deutlicher als im Stuhl. Die Porphyrinausscheidung im Stuhl, insbesondere von Koproporphyrin-Isomer III, bleibt nicht nur in der Remissionsphase noch stark erhöht (Tab. 1), sondern ist auch in der Phase der dekompensierten Latenz (Tab. 4 und Tab. 5; die Untersuchungsproben wurden nicht zu Beginn der klinischen Symptomatik erhalten, sondern nach deren Abklingen) gegenüber der Porphyrinausscheidung im Urin extrem erhöht. Diese Ërgebnisse stehen im Einklang mit den Beobachtungen anderer Autoren $(2,10,12,14)$, aüs denen hervorgeht, daß die exzessive Ausscheidung von Koproporphyrin im Stuhl offensichtlich das führende biochemische Symp: tom der hereditären Koproporphyrie ist. Bèi den hier untersuchten Patienten wurde unsererseits die Diagnose einer hereditären Koproporphyrie aus der Konstellation der Porphyrine und Porphyrinvorläufer im Urin gestellt und durch die nachfolgende Untersuchung der Porphyrinausscheidung im Stühl abgesichert. Von klinischèr Seitè wurde in zwei Fällen (Tab. 1 und 4) der gezielte Verdacht auf eine akute hepatische Porphyrie geäußert, und zwar auf eine akute intermittierende Porphyrie, da das Krankheitśbild der hereditären Koproporphyrie mit dieser analog war. Nur bei einer 
Tab. 4. Hereditäre Koproporphyrie bei einer 4 ljährigen Patientin (M. K.) mit erstmals kolikartigen Schmerzen im Epigastrium, linken Unterbauch und im Rücken. Klinischer Verdacht: Differentialdiagnose zwischen Pankreatitis und akuter intermittierender Porphyrie.

\begin{tabular}{lcc}
\hline & $\begin{array}{l}\text { Urin } \\
(\mathrm{nmol} / 24 \mathrm{~h})\end{array}$ & $\begin{array}{l}\text { Stuhl } \\
(\mathrm{nmol} / \mathrm{g})\end{array}$ \\
\hline Uroporphyrin & 126 & 34 \\
Heptacarboxyporphyrin & 65 & 4 \\
Hexacarboxy porphyrin & 8 & 12 \\
Pentacarboxy porphyrin & 26 & 100 \\
Koproporphyrin & 313 & 2757 \\
Tricarboxyporphyrin & 5 & 10 \\
Dicarboxyporphyrin & 4 & 133 \\
Gesamt-Porphyrine & 547 & 3050
\end{tabular}

$(\mu \mathrm{mol} / 24 \mathrm{~h})$

$\begin{array}{lr}\delta \text {-Aminolävulinsäure } & 40,0 \\ \text { Porphobilinogen } & 0,6\end{array}$

Uroporphyrinogensynthase in den Erythrocyten

$((\mu \mathrm{mol} / \mathrm{h} \cdot 1$ Ery throcy ten (\% der Kontrollen)) 63 (98)

Tab. 5. Hereditäre Koproporphyrie bei einer 22jährigen Patientin (P. U.). Klinische Überlegung: Ausschluß einer Porphyrie.

\begin{tabular}{|c|c|c|}
\hline & $\begin{array}{l}\text { Urin } \\
\text { (nmol/24 h) }\end{array}$ & $\begin{array}{l}\text { Stuhl } \\
\text { (nmol/g) }\end{array}$ \\
\hline $\begin{array}{l}\text { Uroporphyrin } \\
\text { Heptacarboxyporphyrin } \\
\text { Hexacarboxyporphyrin } \\
\text { Pentacarboxyporphyrin } \\
\text { Koproporphyrin } \\
\text { Tricarboxyporphyrin } \\
\text { Dicarboxyporphyrin } \\
\text { (Protoporphyrin) }\end{array}$ & $\begin{array}{r}91 \\
50 \\
13 \\
59 \\
879 \\
3 \\
2\end{array}$ & $\begin{array}{r}7 \\
6 \\
4 \\
23 \\
2276 \\
5 \\
82\end{array}$ \\
\hline Gesamt-Porphyrine & 1097 & 2403 \\
\hline $\begin{array}{l}\text { S-Aminolävulinsäure } \\
\text { Porphobilinogen }\end{array}$ & \multicolumn{2}{|l|}{$\begin{array}{r}45,6 \\
7,6\end{array}$} \\
\hline \multicolumn{3}{|c|}{$\begin{array}{l}\text { Uroporphyrinogensynthase in den Ery throcy ten } \\
((\mu \mathrm{mol} / \mathrm{h} \cdot 1 \text { Erythrocyten (\% der Kontrollen)) } 65 \text { (130) }\end{array}$} \\
\hline
\end{tabular}

Patientin (Tab. 1) bestanden geringgradige, allerdings von der Sonnenexposition abhängige, also für die hereditäre Koproporphyrie nicht typische, Hautsymptome: ein flüchtiges papülöses Exanthem àn Hals und Unterarmen nach Sonnenexposition. Es blieb fraglich, ob diese Symptome mit der hereditären Koproporphyrie ursächlich in Zusammènhang stehen. Die wichtigste „therapeutische" Maßnahme auch für die Patienten mit hereditärer Koproporphyrie ist die prophylaktische Meidung porphyrinogener Medikamente, von Alkohol, Hunger und Streß. Für die therapeutischen Maßnahmen bei hereditärer Koproporphyrie gelten dieselben Regeln, welche für die akute intermittierende Porphyrie erarbeitet wurden (7).
Aus den Erfahrungen der Abgrenzung von Porphyria variegata-Fällen gegenüber der akuten intermittierenden Porphyrie in unserem Krankengut (7) dürfen wir den Schluß ziehen, daß wahrscheinlich eine Reihe „,klinischer" Patienten bzw. Patientinnen mit ,akuter intermittierender Porphyrie" biochemisch der hereditären Koproporphyrie zugeordnet werden müssen, wie dies bereits bei drei Beobachtungen (Tab. 1, 2 und 4) durch die klinisch-chemisch vorgeschlagene Bestimmung des Faecesporphyrinbiochemogramms nach Vorliegen der Urindaten erfolgen konnte. Die praktische Konsequenz der vorliegenden Studie für den klinischen Diagnostiker besteht darin, bei Verdacht auf eine akute hepatische Porphyrie die Metabolite der Porphyrin- und Hämsynthese nicht nur im Urin, sondern auch im Stuhl untersuchen zu lassen und weiterhin die Aktivität der erythrocytären Uroporphyrinogen-Synthase sowohl zur unerläßlichen Sicherung des hereditären Defekts bei akuter intermittierender Porphyrie als auch differentialdiagnostisch zur Abgrenzung der Porphyria variegata und hereditären Koproporphyrie heranzuziehen. Nach den bisherigen Studien liegt das Verhältnis des Vorkommens von akuter intermittierender Porphyrie, Porphyria variegata und hereditärer Koproporphyrie in der Bundesrepublik Deutschland bei 8:1:0,5. Die Aufdeckung der hier vorgestellten Fälle sollte veranlassen, daß bei einer erhöhten Porphyrinausscheidung, insbesondere von Koproporphyrin-Isomer III neben einer nur geringbis mäßiggradig erhöhten Porphyrinvorläufer-Ausscheidung, wobei vor allem auf die erhöhte PorphobilinogenAusscheidung zu achten ist (Abgrenzung gegenüber der akuten Bleiintoxikation), nach einer hereditären Koproporphyrie gefahndet werden muß. Die Porphyrinausscheidung bei hereditärer Koproporphyrie und akuter Bleiintoxikation kann analog sein, jedoch nicht die Porphyrinvorläuferausscheidung: bei der Bleivergiftung sticht die extrem hohe Auscheidung von $\delta$-Aminolävulinsäure gegenüber einer normalen oder nur geringgradig erhöhten Porphobilinogen-Ausscheidung hervor (23). Sollte sich die Differentialdiagnose zwischen akuter Bleivergiftung und hereditärer Koproporphyrie stellen wie sie sich gerade in den letzten Jahren öfters schon zwischen akuter Bleiintoxikation und akuter intermittierender Porphyrie gestellt hat (23) -, dann muß die Fäces-Porphyrin-Ausscheidung als das entscheidende Kriterium herangezogen werden. Eine erhöhte Koproporphyrin-Isomer-III-Ausscheidung im Stuhl wie bei hereditärer Koproporphyrie ist bei der Bleivergiftung nicht bekannt.

Wegen der fließenden biochemischen Úbergänge zwischen den akuten hepatischen Porphyrien hat sich die Bestimmung der Aktivität der UroporphyrinogenSyṇthase als ein zusätzliches differentialdiagnostisches Kriterium bewährt: Die Aktivität dieses Enzyms in den Erythrocyten ist bei hereditärer Koproporphyrie normal. Der in der akuten Phase der metabolischen Krise exzessive Anstieg der Porphyrinvorläufer beruht auf 
der sekundären regulatorischen Kontrollfunktion der hepatischen Uroporphyrinogen-Synthase (,physiologischer Uroporphyrinogen-Synthase-Defekt" $(18,20))$, die insbesondere dann wirksam wird, wenn sich das Substratangebot für das Enzym pathologisch erhöht (5). Möglicherweise ist die Einteilung der akuten hepatischen Porphyrie in drei anscheinend gut definierte Formen in Anbetracht der Variabilität der Gendefekte und Genpenetration, welche letztlich die in der Diagnostik immer wieder neu zu beobachtenden fließenden Ubergänge zwischen den akuten hepatischen Porphyrien bedingen (1), zu willkürlich. Da nicht nur jede Familie ihren eigenen „Porphyrie-Typ“ entwickelt (13), sondern auch innerhalb einer Familie und deren Generationen erhebliche Variationen, z. B. in der Aktivität der Uroporphyrinogen-Synthase, vorkommen können, wird das Konzept der fließenden biochemischen und klinischen Übergänge als Arbeitsgrundlage zur komplexen pathobiochemischen Untersuchung der akuten hepatischen Porphyrien weiter verfolgt: bei den akuten hepatischen Porphyrien spielen nicht nur die Enzymdefekte (21), sondern insbesondere auch deren Kompensation und Gegenregulation eine wesentliche Rolle für die Ausprägung der biochemischen Metabolitkonstellationen (20): die Ausscheidungsprofile sind das Resultat von Enzymdefekt und Kompensation, von Dys- und Gegenregulation (5). Ist der jeweilige primäre partielle Enzymdefekt (21) relativ stark ausgeprägt, kann es zu einer „typischen" klinisch-biochemischen Befundkonstellation für ,akute intermittierende Porphyrie', ,Porphyria variegata' und ,hereditäre Koproporphyrie' kommen; andernfalls, je näher die Enzymaktivität zum Normal- bereich tendiert (Ubberlappungszone), um so fließender und variabler werden die Befundmuster, ohne jedoch die unverwechselbaren Charakteristika für eine akute hepatische Porphyrie zu verlieren (5). Da alle drei akuten hepatischen Porphyrien einen analogen Phasenverlauf (20) aufweisen und auch, akut intermittierend" das akute klinische Syndrom entwickeln, würde der übergeordnete nosologische Begriff ,akute hepatische Porphyrie" klinisch und diagnostisch einfacher sein. und die Krankheit gleichzeitig zutreffender kennzeichnen als die gegenwärtig verwendeten Bezeichnungen: die weitere Differenzierung ist eine vorwiegend biochemische (Metabolitprofile und Enzymdefekt): Typ I mit Uroporphyrinogen-Synthase-Defekt (niemals Hautsymptome), Typ III mit Koproporphyrinogen-OxidaseDefekt (Hautsymptome in Mitteleuropa offensichtlich selten) und Typ III mit Protoporphyrinogen-Oxidaseoder Ferrochelatase-Defekt (Hautsymptome in etwa der Hälfte der Fälle). ,Akut intermittierendes' Auftreten ist durch abdominale, neurologisch-psychische und cardiovaskuläre Symptomatik für jede akute hepatische Porphyrie (Typ I, II und III) charakteristisch.

\section{Danksagung}

Die pathobiochemischen Untersuchungen wurden mit Unterstuitzung der Deutschen Forschungsgemeinschaft durchgefuihrt (Do 134).

Die Marburger Porphyrie-Forschergruppe dankt den Kollegen der am Beginn der Arbeit aufgeführten Kliniken für die Kooperation und das Interesse an der differentialdiagnostischen Problematik der Porphyrien.

Frau Heidrun Heil, Fräulein Ulrike Gilbert und Fräulein Ulrike Kalbfleisch sowie Frau Anna Maria Heusinkveld danken wir für medizinisch-technische Assistenz.

\section{Literatur}

1. Doss, M. \& v. Tiepermann, R. (1978), diese Z. 16, 34-35.

2. Brodie, M. J., Thompson, G. G., Moore, M. R., Beattie, A..D. \& Goldberg, A. (1977), Quart. J. Med., New Series, $X L V I$, Nr. 182, 229-241.

3. Grandchamp, B. \& Nordmann, Y. (1978), in Diagnosis and Therapy of Porphyrias and Lead Intoxication (Doss, M., ed.), Springer, Berlin-Heidelberg-New York.

4. Elder, G. H., Evans, J. O., Thomas, N., Cox, R., Brodie, M. J., Moore, M. R., Goldberg, A. \& Nicholson, D. C. (1976), Lancet II, 1217-1219.

5. Doss, M. (1978), Int. J. Biochem., im Druck.

6. McIntyre, N., Pearsson, A. J., Allan, D. J., Craske, S., West, G. M. L., Moore, M. R., Beattie, A. D., Paxton, J. \& Goldberg, A. (1971), Lancet $I, 561-564$.

7. Doss, M. (1977), Internist 18, 664-675.

8. Doss, M., v. Tiepermann, R., Schmeisser, W., Dölle, W., Höcherl, G., Meinhof, W., Look, D., Henning, H., Vogel, H.-M., Holz, J., Hartmann, W., Goebell, H., Trosse, P., Benker, H. J. \& Geisler, G. (1978), diese Z. 16, 37-37.

9a. Hijmans, A. A. van den Bergh, Regniers, R. \& Muller, P. (1928), Arch. Verdauungskrankh. 42, 302-307.

9b. Watson, C. J., Schwartz, S., Schule, B., Jacobson, L. O. \& Zagaria, R. (1949), J. Clin. Invest. 28, 465-468.
10. Goldberg, A., Rimington, C. \& Lochead, A. C. (1967), Lancet $I, 632-636$.

11. Haeger-Aronsen, B., Stathers, G. \& Swahn, G. (1969), Ann. Int. Med. 69, 221-227.

12. Lomholdt, J. C. \& With, T. K. (1969), Acta Med. Scand. $186,83-85$.

13. With, T. K. (1969), Acta Med. Scand. 186, 117-124.

14. Dean, G. (1972), The porphyrias. A story of inheritance and environment. Pitman, London, pp. 44-53.

15. Nordmann, Y. \& Grandchamp, B. (1978), diese Z. 16, 39-40.

16. Roberts, D. T., Brodie, M. J., Moore, M. R., Thompson, G. G., Goldberg, A. \& MacSween, R. N. M. (1977), Brit. J. Dermatol. 96, 549-554.

17. Doss, M. (1970), diese Z. 8, 197-207.

18. Doss, M. \& Schermuly, E. (1976), In Porphyrins in Human Diseases (M. Doss, ed.) Karger, Basel, pp. 189-204.

19. Schermuly, E. \& Doss, M. (1975), diese Z. 13, 299-304.

20. Doss, M. \& v. Tiepermann, R. (1978), diese Z. 16, 111-118.

21. Doss, M. (1977), Med. Klinik 72, 1501-1518.

22. Doss, M. (1977), Krankenhausarzt 50, 209-21.6.

23. Doss, M. \& v. Tiepermann, R. (1978), diese Z. 16, 57-58.
Prof. Dr. Manfred Doss Fach Klinische Biochemie im Fachbereich Humanmedizin der Philipps-Universität D-355.0 Marburg a. d. Lahn 